\title{
All-in-one interictal presurgical imaging in patients with epilepsy: single-session EEG/PET/(f)MRI
}

\author{
Frédéric Grouiller ${ }^{1} \cdot$ Bénédicte M. A. Delattre ${ }^{1} \cdot$ Francesca Pittau $^{2} \cdot$ Susanne Heinzer $^{3}$. \\ François Lazeyras ${ }^{1}$ - Laurent Spinelli ${ }^{2}$. Giannina R. Iannotti ${ }^{4}$ - Margitta Seeck ${ }^{2}$ • \\ Osman Ratib $^{1}$ - Maria I. Vargas $^{1} \cdot$ Valentina Garibotto $^{1} \cdot$ Serge Vulliemoz $^{2}$
}

Received: 29 October 2014 / Accepted: 10 March 2015 / Published online: 17 April 2015

(C) Springer-Verlag Berlin Heidelberg 2015

\begin{abstract}
Purpose In patients with pharmacoresistant focal epilepsy, resection of the epileptic focus can lead to freedom from seizures or significant improvement in well-selected candidates. Localization of the epileptic focus with multimodal concordance is crucial for a good postoperative outcome. Beyond the detection of epileptogenic lesions on structural MRI and focal hypometabolism on FDG PET, EEG-based Electric Source Imaging (ESI) and simultaneous EEG and functional MRI (EEG-fMRI) are increasingly applied for mapping epileptic activity. We here report presurgical multimodal interictal imaging using a hybrid PET/MR scanner for single-session FDG PET, MRI, EEG-fMRI and ESI.

Methods This quadrimodal imaging procedure was performed in a single session in 12 patients using a highdensity (256 electrodes) MR-compatible EEG system and a hybrid PET/MR scanner. EEG was used to exclude subclinical seizures during uptake of the PET tracer, to compute ESI on interictal epileptiform discharges and to guide fMRI analysis for mapping haemodynamic changes correlated with interictal epileptiform activity.
\end{abstract}

Serge Vulliemoz

Serge.Vulliemoz@hcuge.ch

1 Department of Radiology and Medical Informatics, Geneva University Hospital, 1211 Geneva 14, Switzerland

2 EEG and Epilepsy Unit, Department of Neurology, Geneva University Hospital, Rue Gabrielle-Perret-Gentil 4, 1211 Geneva 14, Switzerland

3 Philips AG Healthcare, 8027 Zürich, Switzerland

4 Functional Brain Mapping Laboratory, Department of Fundamental Neurosciences, Geneva University Hospital, 1211 Geneva 14, Switzerland
Results The whole multimodal recording was performed in less than 2 hours with good patient comfort and data quality. Clinically contributory examinations with at least two modalities were obtained in nine patients and with all modalities in five patients.

Conclusion This single-session quadrimodal imaging procedure provided reliable and contributory interictal clinical data. This procedure avoids multiple scanning sessions and is associated with less radiation exposure than PET-CT. Moreover, it guarantees the same medication level and medical condition for all modalities. The procedure improves workflow and could reduce the duration and cost of presurgical epilepsy evaluations.

Keywords Functional imaging · Hybrid PET/MR . EEG-fMRI $\cdot$ Epilepsy surgery

\section{Introduction}

In patients suffering from pharmacoresistant epilepsy who are candidates for epilepsy surgery, the success of the surgery relies on the presurgical work-up that aims to localize the epileptic focus and determine its proximity to eloquent cortical regions (e.g. language, sensorimotor and visual cortex). Presurgical evaluations are based on long-term video-EEG and structural MRI, often complemented by interictal FDG PET and ictal SPECT. In addition, other noninvasive tools such as EEG-based electric source imaging (ESI) and simultaneous EEG and functional MRI (EEG-fMRI) are increasingly used in many centres for the functional localization of epileptic focus.

Structural lesions seen on MRI acquired during an epilepsy-dedicated MRI protocol and reviewed by a neuroradiologist with expertise in epilepsy imaging clearly improve 
the odds of successful surgery $[1,2]$. Currently, MRI remains negative in $20-30 \%$ of patients evaluated for epilepsy surgery [3]. In doubtful or non-lesional cases, morphometric and quantitative analyses of structural MRI may help detecting subtle forms of focal cortical dysplasia [4].

FDG-PET reveals areas of interictal cerebral hypometabolism associated with epileptic activity and epileptogenic lesions $[5,6]$. It is particularly useful in patients with apparently nonlesional epilepsy $[5,7]$. In particular instances of multifocal epilepsy, the combination of PET and other imaging techniques or the use of specific tracers such as alphamethyl-tryptophan for tuberous sclerosis could improve localization [8-10]. EEG monitoring before and during uptake of the PET tracer is useful for detecting subclinical epileptic seizures that could cause false-negative results by confounding interictal hypometabolism with ictal hypermetabolism [6].

Functional localization of the epileptic activity can be performed with electrophysiological techniques [11]. EEG-based ESI is both sensitive and specific in estimating the localization of the cortical generators of epileptic activity in lesional and nonlesional epilepsy, particularly when high-density EEG systems are used [12]. Its clinical relevance is supported by the fact that the noninvasive localization of interictal spikes with ESI provides a good estimate of the seizure onset zone (measured with intracranial EEG recordings [13]) and the epileptogenic zone (estimated by resection volumes and postoperative follow-up [12]). The simultaneous recording of EEG and fMRI (EEG-fMRI) allows mapping of haemodynamic changes related to interictal epileptic activity. Advanced analyses can reliably detect haemodynamic fluctuations even in the absence of epileptic spikes during fMRI, as confirmed by invasive validation and result in an important increase in the technique's sensitivity [14]. In many difficult cases, the EEEG-fMRI results add information to the presurgical workup [15]. They are concordant with invasive EEG recordings $[14,16]$ and can predict postoperative outcome [17].

The localization of the epileptic focus relies, besides clinical and neuro-psychological data, on these structural and functional brain imaging methods. Concordance of the multimodal imaging results is associated with a better postoperative outcome and is the key factor for tailoring surgical resection or deciding whether an invasive EEG recording with intracranial electrodes is needed to better map the epileptic focus and the eloquent cortex.

In our centre, structural MRI, PET, ESI and EEG-fMRI are routinely performed in separate sessions in the context of the presurgical evaluation. Hybrid PET/MR systems have recently been available for clinical applications [18] and the combination of a hybrid scanner with MR-compatible EEG equipment offers a very interesting opportunity to obtain data for structural MRI, EEG-monitored PET, EEG-fMRI and ESI in the same session. In this way, the long-term video-EEG recording is minimally interrupted and direct integration of structural, metabolic, haemodynamic and electrophysiological signatures of the disease can be obtained with the same medication level.

We here report, for the first time, combined EEG-PET, MRI, EEG-fMRI and ESI based on data recorded in a single session using a high-density EEG and a PET-MR hybrid scanner in 12 patients with pharmacoresistant epilepsy.

\section{Materials and methods}

\section{Patients}

For this single-session quadrimodal imaging, we selected patients investigated in our presurgical evaluation unit for drugresistant focal epilepsy. We recruited 12 consecutive patients (median age at evaluation 33 years, range $10-56$ years, eight female) who, according to referral letters, had reports of interictal spikes on routine EEG and were cognitively able to tolerate a 2-h imaging session. A high interictal spiking rate was not a necessary criterion and patients with rare interictal epileptiform discharges (IEDs) were also included.

All adult patients and the parents of paediatric patients gave written informed consent for the EEG-fMRI as part of our EEG-fMRI research programme approved by the local ethics committee. All other tests were part of the routine clinical work-up in our centre.

\section{Equipment}

High-density EEG

EEG was recorded at $1 \mathrm{kHz}$ using a 256-channel MR-compatible system with a high input impedance amplifier (Electrical Geodesics Inc., Eugene, OR).

\section{Hybrid PET/MR}

Patients were scanned using an Ingenuity TF PET-MR scanner (Philips Healthcare, Cleveland, $\mathrm{OH}$ ). This system combines a 3-T MRI scanner with multiple transmission capabilities (Achieva 3.0T TX series) and a PET scanner equipped with time-of-flight technology. The two elements are linked via a single patient table allowing sequential imaging with both modalities in the same frame of reference by a $180^{\circ}$ rotation of the scanner table between the MRI and the PET scanner. All acquisitions (PET-EEG, structural MRI and EEGfMRI) were done with this hybrid PET-MR scanner.

\section{Procedure}

A flow chart showing the multimodal integration procedure is presented in Fig. 1. 


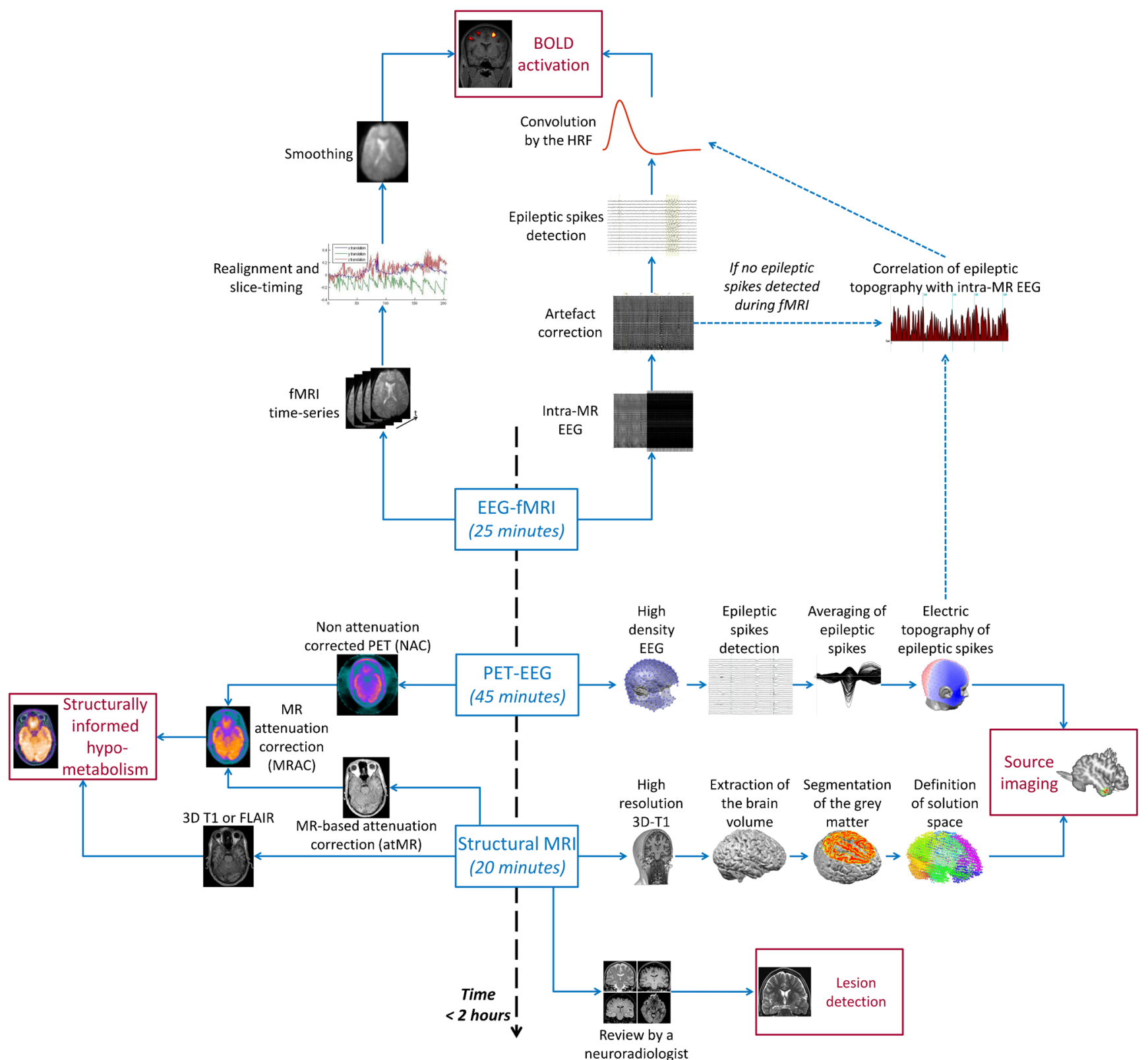

Fig. 1 Multimodal integration procedure. EEG-fMRI, PET-EEG and structural MRI were acquired during the same session. The results of the four different modalities (epilepsy-related BOLD activation, EEG

\section{Installation of EEG net outside the scanner room $(15 \mathrm{~min})$}

The EEG net was adjusted according to the international 10/ 10 system. The 256-electrode sensor net guaranteed full coverage of the head with a constant intersensor distance. The structure of the net ensured that the electrodes were evenly distributed over the scalp and that they were positioned at approximately the same location across patients. Each electrode contains a small sponge soaked with saline water that touches the patient's scalp surface directly. For safety reasons, each electrode is equipped with an additional $10 \mathrm{k} \Omega$ resistance [19]. Electrode impedances were kept as low as possible source imaging, structural-informed PET hypometabolism and lesion detection on structural MRI) were then used to identify the potential target for surgery

(ideally below $30 \mathrm{k} \Omega$ ) in order to guarantee high-quality EEG [20].

\section{EEG-fMRI recordings (25 $\mathrm{min})$}

The patients were installed in the MRI scanner with their head carefully immobilized while ensuring optimal comfort using memory foam pillows. EEG and fMRI were acquired simultaneously and continuously for $20 \mathrm{~min}$ while the patient was at rest with eyes closed. EEG acquisition was synchronized with the MRI clock to facilitate removal of gradient artefacts [21]. A total of 600 functional images were acquired with an eight- 
channel head coil using a T2*-weighted single-shot gradient echo echo-planar imaging sequence (TR 1,984 ms, TE $30 \mathrm{~ms}$, voxel size $1.78 \times 1.78 \times 3 \mathrm{~mm}, 32$ slices).

\section{FDG PET with EEG monitoring (45 min)}

A 15 -min ${ }^{18} \mathrm{~F}$-FDG PET acquisition was performed $30 \mathrm{~min}$ after intravenous injection of the tracer. During uptake of the tracer, the patients were at rest and the EEG was recorded simultaneously to ensure that the patient was in an interictal state and to document potential subclinical epileptic seizures that could have modified metabolism and alter the interpretation. Our protocol required the patient to remain in a quiet environment with eyes closed during the uptake period to avoid spurious hypermetabolism in the auditory or visual cortices. The simultaneous acquisition of functional and structural images was therefore not possible during tracer uptake. The EEG net was removed before PET scanning to avoid the potential effect of the electrodes on the PET images and to maximize patient comfort.

\section{Structural MRI (20 min)}

After rotation of the patient table, an epilepsy-specific MRI protocol was acquired in coregistration with the PET acquisition. We obtained high-resolution 3D T1 structural images and, if not yet available from a recent clinical scan, 3D FLAIR, coronal T2 and diffusion imaging were also performed. For PET attenuation correction, a fast 3D gradientecho $\mathrm{T} 1$ sequence was also acquired in all patients (MR-based attenuation correction) [22].

\section{Analyses}

\section{ESI}

EEG acquired at rest during FDG tracer uptake was reviewed by an experienced neurophysiologist (S.V. or F.P.) to exclude subclinical seizures and to mark IEDs. Artefact-free and isolated IEDs were averaged within a window of $500 \mathrm{~ms}$ before and after the IED peak [12]. Artefact-affected channels were interpolated using the signal from neighbouring electrodes [23].

To compute the forward model, we used a simplified realistic head model based on the individual patient's highresolution 3D T1-weighted MRI images. After extraction and segmentation of the brain, 5,000 solution points were distributed at regular distances within the grey matter. A locally Spherical Model with Anatomical Constraints [24] was used with standardized electrode positions to calculate the lead field. A linear distributed inverse solution algorithm with biophysical constraints was used to estimate the 3D current density distribution [25]. This simplified anatomically constrained head model has similar localization precisions to a realistic boundary element model [26] and has been successfully used in several clinical studies [27, 28, 12, 29, 30]. The EEG map at the $50 \%$ rising phase of the averaged IEDs was considered for source analysis, as this time-point more accurately localizes the underlying electrical source than the peak which already involves areas of spike propagation [31]. All EEG analyses were carried out using the freely available software Cartool (https://sites.google.com/site/fbmlab/cartool) [24].

\section{EEG-fMRI}

EEG processing Gradient and pulse-related artefacts were removed using a moving average template subtraction method $[32,33]$. If necessary residual artefacts (i.e. cardiac artefacts, eye-blinks, muscular activity, scanner-related residuals) were removed using a temporal independent component analysis [34].

An experienced neurophysiologist (S.V. or F.P.) manually detected IEDs. If few or no spikes were found during the 20 min of simultaneous recording, fMRI analysis was guided by EEG topography analysis following our previous work [14]: the patient-specific epileptic topographic map obtained during the PET tracer uptake was correlated at each time-point with the intra-MR EEG to estimate the presence of the pathological map.

The IED timing or the time-course of the topography-based correlation was then convolved with the canonical haemodynamic response function and used as a regressor in the fMRI analysis.

fMRI processing The spatial preprocessing of functional images was performed using SPM8 (Wellcome Department of Imaging Neuroscience, UCL, London, UK) and included slice-timing correction, realignment of functional images, spatial smoothing with an isotropic Gaussian kernel of $6 \times 6 \times 6 \mathrm{~mm}^{3}$ (full-width at half-maximum) and coregistration of realigned images on the 3D T1 structural MRI images. fMRI time series were whitened and serial correlations were removed using an autoregressive filter of order 1. Low-frequency noise and signal drift were removed using a discrete cosine transform basis set with a filter cut-off period of $128 \mathrm{~s}$. A general linear model was built including the regressor derived from the spike-based analysis or from the topographical analysis as the effect of interest and the six motion-related parameters derived from the functional images realignment as covariates.

For each patient, paired $t$-tests were applied at each voxel to test for BOLD increases and decreases associated with the epileptic regressor. The significance level was set to $p<0.001$ with a threshold extent of five voxels to discard scattered activations suggestive of noise. This analysis procedure was the same as in our previous EEG-fMRI work in 
which results were validated by subsequent intracranial EEG or surgical outcome [14].

\section{PET}

High-resolution $(2 \times 2 \times 2 \mathrm{~mm}$ voxel size $)$ reconstruction was performed using the coregistered MR-based attenuation correction map. After fusion with the high-resolution 3D MRI structural image, areas of hypometabolism were visually identified by an expert in nuclear medicine (V.G.).

\section{Structural MRI}

Structural abnormalities were detected visually by a neuroradiologist with expertise in epilepsy imaging (M.I.V.). For each patient, we considered the result of each method by integration of multimodal information. The results of each modality were considered contributory if they provided localization information concordant with the electroclinical semiology as defined by a consensus of our clinical multidisciplinary case discussion panel. We considered that examinations discordant with each other but concordant with the electroclinical semiology were also clinically contributory as they might have been highlighting different aspects of an epileptic network (e.g. propagation pattern) in specific situations.

\section{Results}

The whole procedure was performed in less than 2 hours of scanner time and was well tolerated but could not be fully completed in two patients. One examination in a 10-year-old patient with behavioural problems (patient 9) needed to be interrupted during the inaugural EEG-fMRI due to excessive motion and noncompliance from the beginning of the procedure. The EEG could not be acquired during fMRI and uptake of the PET tracer in one patient (patient 5) due to a technical problem with the EEG acquisition system.

A lesion was identified on the structural MRI images in 7 of 11 patients and focal hypometabolism was detected in 8 of 11 patients on PET. IEDs were detected in 8 of 11 patients allowing electric source localization. BOLD changes associated with interictal epileptic activity were obtained in 7 of 11 patients. In two patients (patients 3 and 6), IEDs were identified during fMRI recording. In the other five patients (patients 1, 2, 4, 7 and 12), the epileptic topographic map was used to perform the EEG-fMRI analysis. Table 1 summarizes the results for each modality in the 12 patients. Overall, of the four examinations, four were contributory in five patients, two were contributory in four patients and one was contributory in two patients. In one patient, all tests were negative.

Results of the multimodal imaging procedure in three example patients are presented in Figs. 2, 3 and 4. Patient 7
(Fig. 2) had a right frontal transmantle cortical dysplasia visible on the structural MRI images. The epileptogenicity of this lesion was confirmed by hypometabolism on interictal PET images, the EEG source localization and epilepsy-related BOLD changes. Patient 3 (Fig. 3) had non lesional right temporal epilepsy. Concordant ESI and EEG-fMRI results in the right anterior temporal lobe were confirmed by intracranial electrodes. Patient 6 (Fig. 4) had non lesional structural MRI images and no detectable hypometabolism on interictal PET images. Diffuse bilateral frontal IEDs with maximal amplitude on the right side were detected in the high-resolution EEG during FDG uptake and simultaneous EEG-fMRI. The source of this activity was located in the middle and inferior right frontal gyri, and quickly propagated to the contralateral area. BOLD changes related to these IEDs were concordant with the spike field. These multimodal findings supported the diagnosis of right frontal nonlesional epilepsy with secondary bilateral synchrony.

\section{Discussion}

In this study we demonstrated for the first time the feasibility and clinical usefulness of recording PET, MRI, EEG-fMRI and ESI in a single session. This quadrimodal imaging procedure offers a valuable tool for optimizing the workflow of the presurgical work-up with a single-session interictal imaging that minimizes radiation exposure (no PET-CT) and involves only a single excursion outside the EEG telemetry ward.

\section{Clinical considerations}

As shown by our results, a perfect total concordance between all modalities was rarely achieved in this group. This reflects the fact that epilepsy patients seen at most epilepsy surgery centres are difficult cases with a high proportion having no structural lesion (4 of 11), rare interictal events (3 of 11) and discordance between structural MRI and EEG. The combination of multiple imaging techniques is therefore of paramount importance in this patient population to compensate for the high proportion of negative examinations and to increase the reliability of positive findings when modalities are concordant. The multimodal session can yield single or multiple, concordant or discordant clinically meaningful localizing features that altogether contribute to guide patient management. The sensitivity of the different techniques for localizing structural or functional abnormalities appears to be comparable to those found in larger single modality studies. Importantly, the finding of apparently discordant localization for different techniques might fit well within the multimodal findings related to specific epileptic conditions, and results should always be interpreted in the light of the clinical context. For instance, anterior temporal localization of interictal activity is concordant with the known 


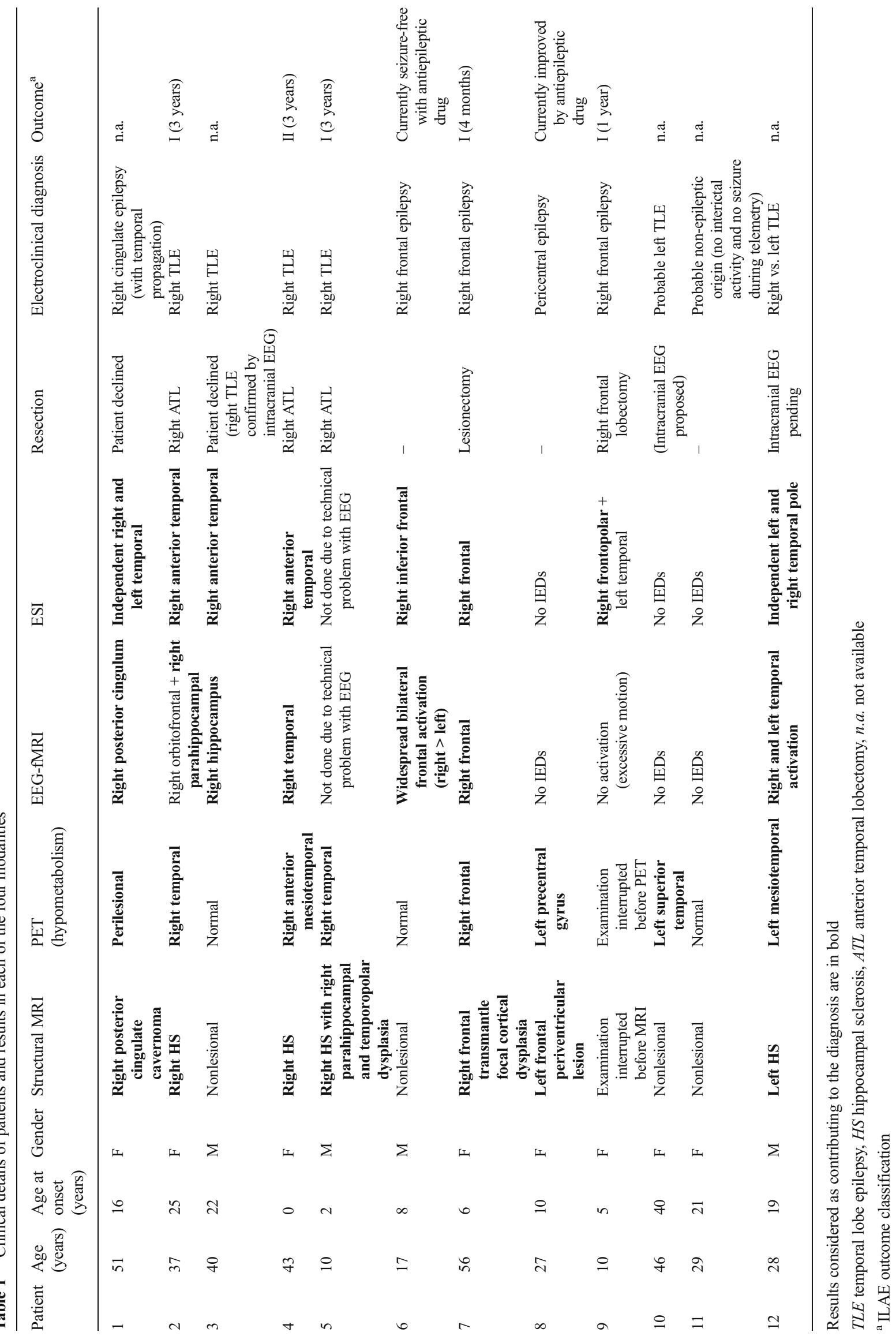


(a) MRI

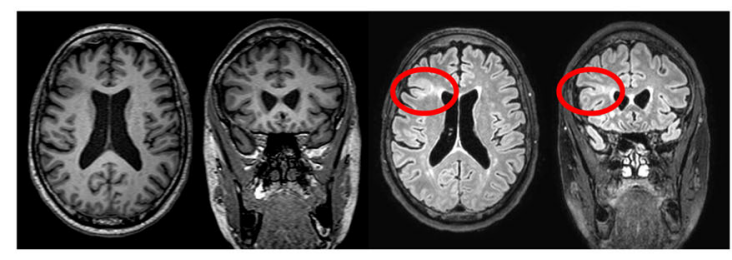

(c) ESI
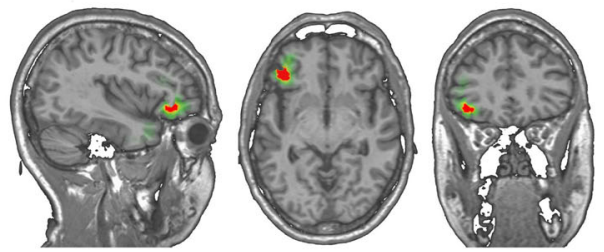

Fig. 2 Multimodal imaging in patient 7 (with lesional right frontal epilepsy). a Right frontal transmantle cortical dysplasia is apparent on the structural images (left T1; right FLAIR, lesion is highlighted by the red circles). $\mathbf{b}$ An extended zone of hypometabolism, encompassing the lesion, is apparent in the right frontal lobe on interictal PET images (b) PET

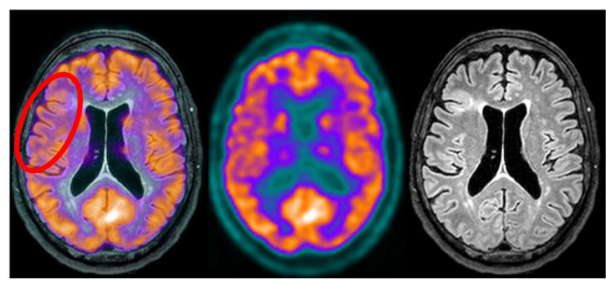

(d) EEG-fMRI
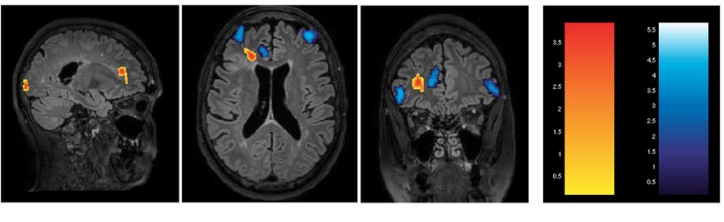

(hypometabolism is highlighted by the red circle). $\mathbf{c}$ EEG source and $\mathbf{d}$ topography-related fMRI activation are located in right prefrontal cortex (fMRI activation in red, deactivation in blue) in close proximity to the lesion. The images are presented in the radiological convention (right hemisphere on the left of the image)

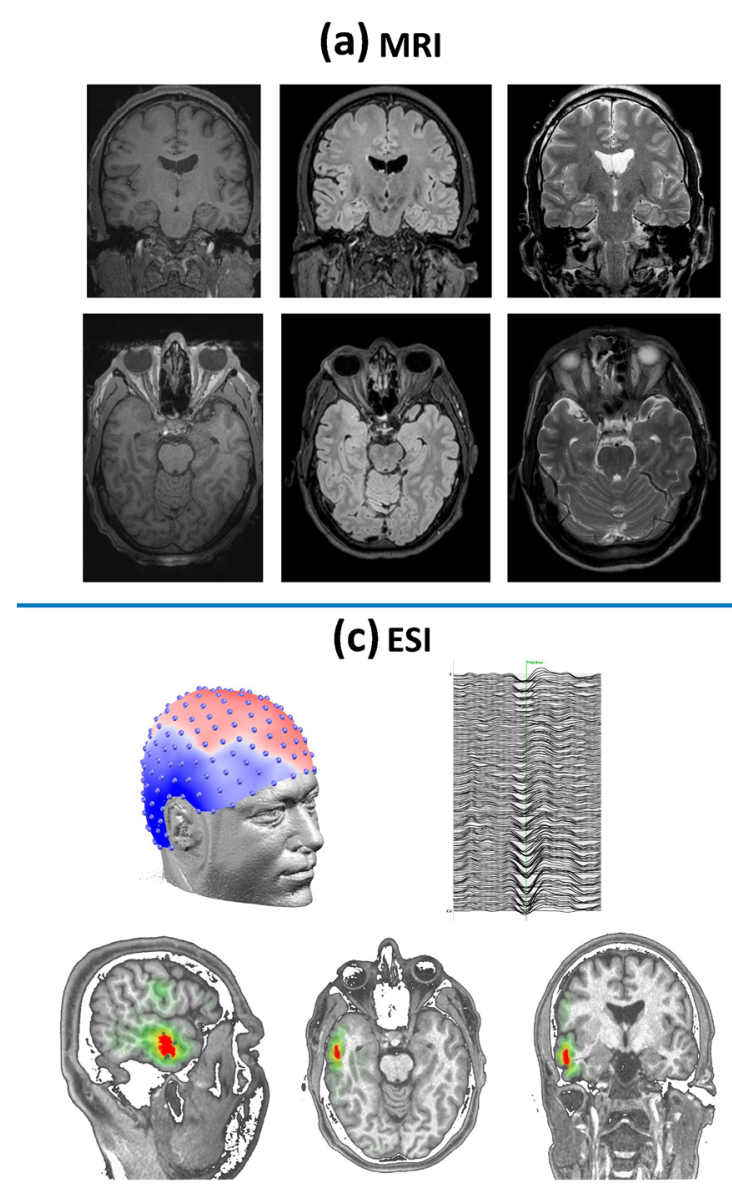

Fig. 3 Multimodal imaging in patient 3 (with non lesional mesial temporal lobe epilepsy). a No lesion is apparent on the T1 (left), FLAIR (middle) and T2 (right) structural MRI images. b No significant focal hypometabolism is apparent on the PET images. c EEG source is

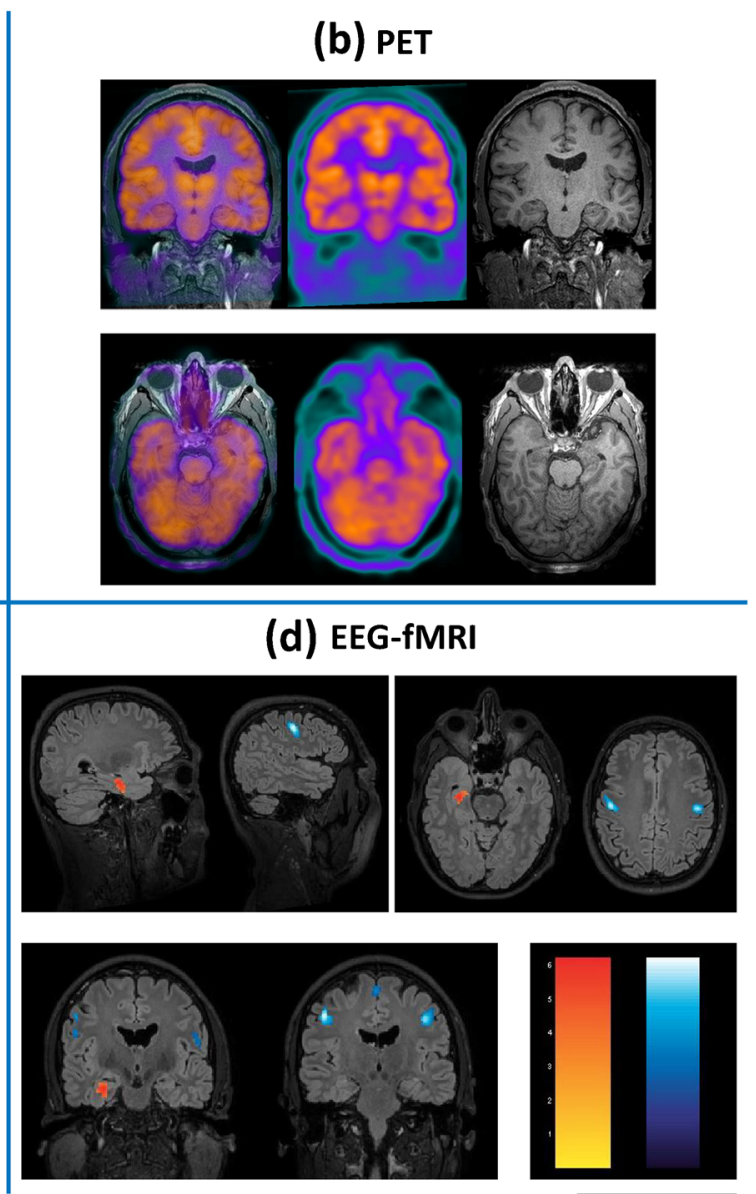

located in the right temporal pole while $\mathbf{d}$ IED-related fMRI activation is apparent in the right hippocampus (fMRI activation in red, deactivation in blue). The images are presented in the radiological convention (right hemisphere on the left of the image) 
(a) MRI
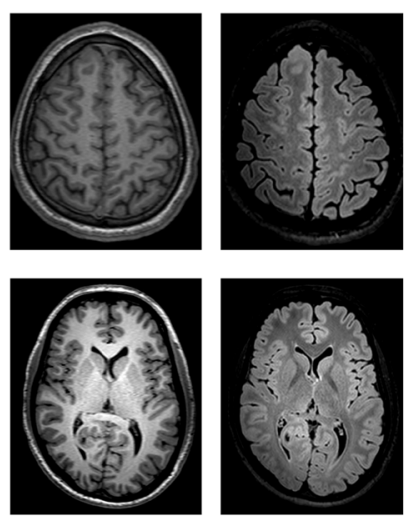

(c) ESI
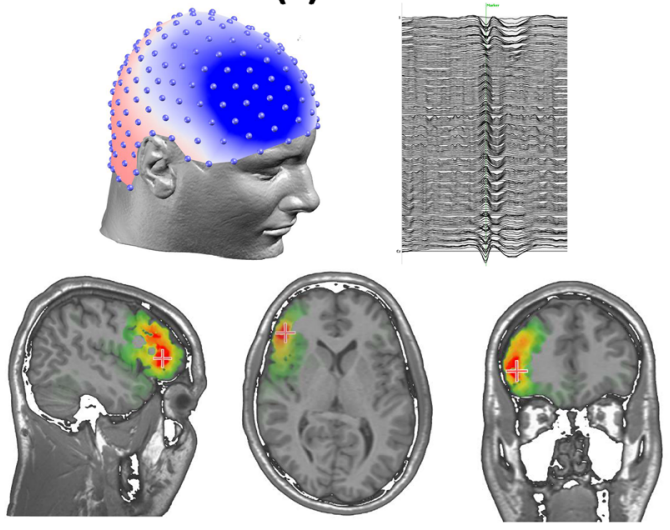

Fig. 4 Multimodal imaging in patient 6. a No lesion is apparent on the T1 (left), FLAIR (middle) and T2 (right) structural MRI images. b No focal hypometabolism is apparent on the PET images. c EEG source imaging locates the epileptic source in the right middle and inferior

propagation patterns from posterior cingulate lesions and does not contradict the structural findings (patient 1) [35]. In rare difficult diagnostic cases such as patient 11, negative results in all imaging tests combined with the absence of epileptic activity or seizures during telemetry supported the final hypothesis of probable non-epileptic attacks.

\section{Integration of PET and MRI}

The advantages of hybrid PET/MR technology have previously been addressed $[36,18]$. PET and MRI coregistration has already proven its importance especially in the identification of subtle epileptic lesions [37]. Although abundantly and routinely performed by brain imaging research groups, the fusion of images acquired on separate systems is not always straightforward and is hardly compatible with the clinical and security constraints imposed by medical image archiving systems. To fully benefit from the hybrid PET/MR system, the patient's head should not move between the structural MRI and the PET acquisition. The long total duration of the protocol increases the likelihood of major head motion. In our procedure, (b) PET
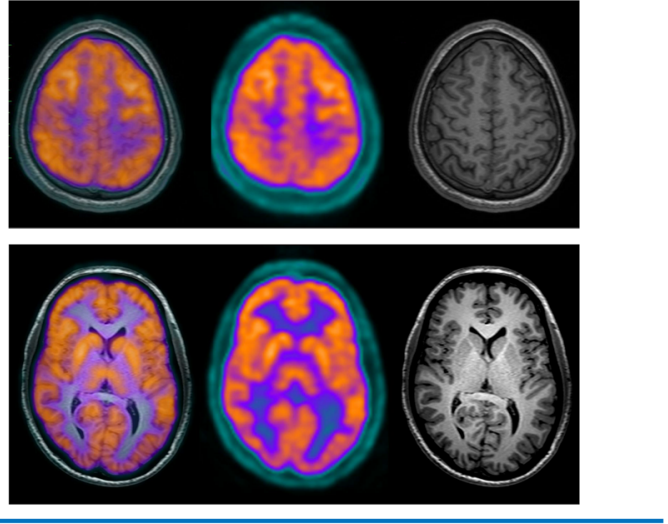

(d) EEG-fMRI
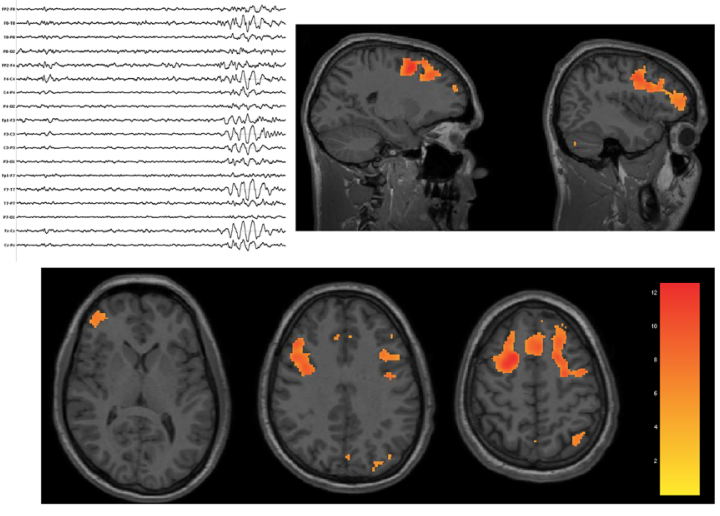

frontal gyri. d IEDs recorded using EEG during fMRI show a strong bilateral frontal activation maximal on the right. The images are presented in the radiological convention (right hemisphere on the left of the image)

the removal of the EEG cap just before PET and structural MRI acquisition allows the patient to move slightly and readjust the position of the head for optimal comfort. Structural MRI was acquired just after the PET acquisition ensuring that PET and structural MRI were acquired in exactly the same position. In the end, only the fMRI images were not acquired in the same reference as the rest of the examination but the standard preprocessing of functional images in fMRI analysis includes realignment and coregistration with the structural MRI images to reduce the effect of intrasession head motion.

MR-based attenuation correction is a widely debated matter. MR does not provide a map of the attenuation coefficient of the tissue, as opposed to CT. For this reason alternative strategies (segmentation of the MR image, use of reference atlases) have been developed [38]. The solution adopted in this study is shared by the hybrid PET/MR scanners currently in use, is clinically approved and represents the current standard [39]. Despite the fact that various approaches have been tested, no ideal solution is available yet, and some of the improved techniques which have been specifically developed to overcome this problem introduce other systematic biases 
[40]. For this reason, no quantitative analysis (statistical comparison with a reference database acquired on PET-CT or ratio between regions) was used in this study: we based the interpretation of the images on the visual evaluation of focal hypoactivities relative to the adjacent cortex, which is not influenced by the correction strategy adopted. On the other hand, MR-based attenuation correction has the advantage of avoiding the radiation exposure related to the $\mathrm{CT}$ acquisition currently used in PET-CT scans. This could be of even greater interest in the paediatric population and in patients requiring repeated investigations.

\section{Single session of multimodal imaging}

During the presurgical evaluation of epileptic patients, PET, structural MRI, EEG-fMRI and high-density EEG recordings are usually done in four separate sessions. The ability to record all these modalities in a single session allows a considerable decrease in the total examination duration. It avoids multiple interruptions in long-term video-EEG monitoring and eases the scheduling of these examinations especially in busy clinical PET-CT and MRI units. This integrated interictal imaging work-flow does not reduce the critical need for ictal data using video-EEG telemetry and, if available, ictal SPECT which provides invaluable complementary information [5]. In our clinical setting, patients are referred from other hospitals and it is highly beneficial to perform the whole work-up (multimodal imaging and telemetry) during the same hospital stay to minimize patient travel and accelerate clinical decisions. It could also consequently reduce the duration and costs of presurgical evaluation. In compliant patients, an additional short fMRI protocol for localizing eloquent cortical areas could be added (motor, sensory, language areas) [41, 42]. The youngest patient already showed marked anxiety and restlessness during installation in the scanner leading to abortion of the whole procedure after a few minutes of scanning, and the same limitations would probably have been encountered with separate sessions of unsedated imaging in this patient. To extend the applicability of this single session of multimodal imaging in very young patients or those with behavioural problems, sedation could be applied if needed for PET and structural MRI as frequently performed in our centre for independent PET-CT and MRI acquisitions. Importantly, sedation should be administered at the end of tracer uptake, just prior to PET acquisition, in order to avoid interference between sedation and brain metabolism during uptake. Sedation for EEG-fMRI can alter epileptic activity and in our presurgical paediatric population, we routinely acquire EEGfMRI without sedation. However, EEG-fMRI under light sedation can be performed successfully [43] although our current protocol should be changed to avoid interference of EEGfMRI sedation with the subsequent PET tracer uptake.
Multimodal image acquisition in the same session also guarantees that disease activity and treatment effect are as similar as possible. This is not necessarily the case for separate acquisitions, which are often performed days if not weeks apart. This may be relevant to comparison of functional and metabolic imaging such as PET, EEG-fMRI and ESI, notably with regard to the extent of the areas involved in IEDs which can be heavily influenced by medication and the multimodal approach offers invaluable data for studying the relationships between metabolism, haemodynamics and neuroelectrical activity without the confounding effects of treatment difference.

The installation and comfort of the patient deserve careful attention to avoid increased motion towards the end of the procedure. The head of the patient was carefully immobilized to guarantee coregistration of the different modalities and keep the same reference between PET and MRI. A memory foam pillow noticeably improved the comfort of the patient with respect to the potential pressure of the EEG electrodes on the back of the head. After the EEG recording during EEGfMRI and uptake of the PET tracer, we removed the EEG cap. This ensured the same head position between the PET scan and the MRI structural protocol and allowed the recording of a full and high-quality structural MR epilepsy protocol that included sequences with a high specific absorption rate (i.e. FLAIR) that are not recommended with the MR-conditional EEG cap due to the risk of heating [44].

\section{EEG-fMRI}

In this study only 2 of 11 patients had enough interictal epileptiform events during simultaneous EEG-fMRI for a conventional "IED-correlated" analysis. The high proportion of patients with rare or no spikes during EEG-fMRI could have been due to our inclusion criteria that were not restricted to patients with a high interictal spiking rate. The duration of EEG-fMRI scanning (20 min, 600 volumes) was similar to that used in our previous studies and our usual EEG-fMRI protocol, although some others acquire much longer datasets to increase the number of detected IEDs. Among the nine patients without IEDs during EEG-fMRI, three had very rare interictal events even outside the MRI and would probably not have been suitable for spike-correlated EEG-fMRI. The detection of more frequent interictal events in the EEG recorded during uptake of the PET tracer is more likely because the patient was at rest without any auditory stimulation (no acquisition) and the EEG was not subject to MR-related artefacts. Our topography-related method does not require IEDs to map the epileptic network using EEG-fMRI [14] and showed clinically meaningful results in five patients.

Electrode-related imaging artefacts caused by local modification of the magnetic susceptibility were limited to the scalp and skull and the BOLD fMRI signal was not significantly affected, in concordance with a previous report [45]. The 
presence of electrode-related artefacts could be of great interest for the individual localization of each electrode for ESI [46], but our current protocol did not include a T1-weighted image with the EEG cap to avoid increasing the total session duration.

\section{Conclusion}

The whole multimodal recording could be performed in less than 2 hours of scanner time with good patient comfort. This single-session quadrimodal imaging provided reliable interictal clinical data with reduced total scanning time. This procedure avoids multiple scanning sessions, reduces irradiation compared to the use of PET-CT, simplifies the coregistration of the results from the different modalities and ensures that the patient is in the same disease state and under the same medication. Therefore, this new multimodal procedure with hybrid PET/MRI and high-resolution EEG may considerably improve the workflow in presurgical epilepsy and could reduce the cost and duration of presurgical evaluations.

Acknowledgments This work was supported by the Swiss National Science Foundation for Scientific Research (grant nos. 33CM30140332 and 320030-141165 and 146633), the Gertrude von Meissner Foundation and by the Centre for Biomedical Imaging (CIBM) of the Universities and Hospitals of Geneva and Lausanne, and the EPFL.

Conflicts of interest S.H. and B.D. are Philips clinical scientists hired to run the scanner together with the hospital staff. They provided valuable technical assistance and are included in the authors' list. However, the data analysis and interpretation were performed by the authors with pure academic affiliations with complete independence and without any conflicts of interest with respect to Philips Healthcare. The industrial company had no influence on the writing of this paper. The remaining authors have no conflicts of interest.

\section{References}

1. Spencer S, Huh L. Outcomes of epilepsy surgery in adults and children. Lancet Neurol. 2008;7(6):525-37.

2. Von Oertzen J, Urbach H, Jungbluth S, Kurthen M, Reuber M, Fernandez G, et al. Standard magnetic resonance imaging is inadequate for patients with refractory focal epilepsy. J Neurol Neurosurg Psychiatry. 2002;73(6):643-7.

3. Duncan JS. Imaging in the surgical treatment of epilepsy. Nat Rev Neurol. 2010;6(10):537-50.

4. Wagner J, Weber B, Urbach H, Elger CE, Huppertz HJ. Morphometric MRI analysis improves detection of focal cortical dysplasia type II. Brain J Neurol. 2011;134(Pt 10):2844-54.

5. Chassoux F, Rodrigo S, Semah F, Beuvon F, Landre E, Devaux B, et al. FDG-PET improves surgical outcome in negative MRI Taylor-type focal cortical dysplasias. Neurology. 2010;75(24): 2168-75.

6. Van Paesschen W, Dupont P, Sunaert S, Goffin K, Van Laere K. The use of SPECT and PET in routine clinical practice in epilepsy. Curr Opin Neurol. 2007;20(2):194-202.
7. Carne RP, O'Brien TJ, Kilpatrick CJ, MacGregor LR, Hicks RJ, Murphy MA, et al. MRI-negative PET-positive temporal lobe epilepsy: a distinct surgically remediable syndrome. Brain J Neurol. 2004;127(Pt 10):2276-85.

8. Kagawa K, Chugani DC, Asano E, Juhasz C, Muzik O, Shah A, et al. Epilepsy surgery outcome in children with tuberous sclerosis complex evaluated with alpha-[11C]methyl-L-tryptophan positron emission tomography (PET). J Child Neurol. 2005;20(5):429-38.

9. Kargiotis O, Lascano AM, Garibotto V, Spinelli L, Genetti M, Wissmeyer M, et al. Localization of the epileptogenic tuber with electric source imaging in patients with tuberous sclerosis. Epilepsy Res. 2014;108(2):267-79.

10. Kumar A, Juhasz C, Asano E, Sood S, Muzik O, Chugani HT. Objective detection of epileptic foci by 18F-FDG PET in children undergoing epilepsy surgery. J Nucl Med. 2010;51(12):1901-7.

11. Pittau F, Grouiller F, Spinelli L, Seeck M, Michel CM, Vulliemoz S. The role of functional neuroimaging in pre-surgical epilepsy evaluation. Front Neurol. 2014;5:31.

12. Brodbeck V, Spinelli L, Lascano AM, Wissmeier M, Vargas MI, Vulliemoz S, et al. Electroencephalographic source imaging: a prospective study of 152 operated epileptic patients. Brain J Neurol. 2011;134(Pt 10):2887-97.

13. Megevand P, Spinelli L, Genetti M, Brodbeck V, Momjian S, Schaller K, et al. Electric source imaging of interictal activity accurately localises the seizure onset zone. J Neurol Neurosurg Psychiatry. 2014;85(1):38-43.

14. Grouiller F, Thornton RC, Groening K, Spinelli L, Duncan JS, Schaller K, et al. With or without spikes: localization of focal epileptic activity by simultaneous electroencephalography and functional magnetic resonance imaging. Brain J Neurol. 2011;134(Pt 10):2867-86.

15. Pittau F, Dubeau F, Gotman J. Contribution of EEG-fMRI to the definition of the epileptic focus. Neurology. 2012;78(19):1479-87.

16. Thornton R, Vulliemoz S, Rodionov R, Carmichael DW, Chaudhary UJ, Diehl B, et al. Epileptic networks in focal cortical dysplasia revealed using electroencephalography-functional magnetic resonance imaging. Ann Neurol. 2011;70(5):822-37.

17. Thornton R, Laufs H, Rodionov R, Cannadathu S, Carmichael DW, Vulliemoz S, et al. EEG correlated functional MRI and postoperative outcome in focal epilepsy. J Neurol Neurosurg Psychiatry. 2010;81(8):922-7.

18. Garibotto V, Heinzer S, Vulliemoz S, Guignard R, Wissmeyer M, Seeck M, et al. Clinical applications of hybrid PET/MRI in neuroimaging. Clin Nucl Med. 2013;38(1):e13-8.

19. Lemieux L, Allen PJ, Franconi F, Symms MR, Fish DR. Recording of EEG during fMRI experiments: patient safety. Magn Reson Med. 1997;38(6):943-52.

20. Ferree TC, Luu P, Russell GS, Tucker DM. Scalp electrode impedance, infection risk, and EEG data quality. Clin Neurophysiol. 2001;112(3):536-44.

21. Mandelkow H, Halder P, Boesiger P, Brandeis D. Synchronization facilitates removal of MRI artefacts from concurrent EEG recordings and increases usable bandwidth. Neuroimage. 2006;32(3): $1120-6$.

22. Schulz V, Torres-Espallardo I, Renisch S, Hu Z, Ojha N, Bornert P, et al. Automatic, three-segment, MR-based attenuation correction for whole-body PET/MR data. Eur J Nucl Med Mol Imaging. 2011;38(1):138-52.

23. Perrin F, Pernier J, Bertrand O, Echallier JF. Spherical splines for scalp potential and current density mapping. Electroencephalogr Clin Neurophysiol. 1989;72(2):184-7.

24. Brunet D, Murray MM, Michel CM. Spatiotemporal analysis of multichannel EEG: CARTOOL. Comput Intell Neurosci. 2011;2011:813870.

25. Grave de Peralta Menendez R, Murray MM, Michel CM, Martuzzi $\mathrm{R}$, Gonzalez Andino SL. Electrical neuroimaging based on biophysical constraints. Neuroimage. 2004;21(2):527-39. 
26. Guggisberg AG, Dalal SS, Zumer JM, Wong DD, Dubovik S, Michel CM, et al. Localization of cortico-peripheral coherence with electroencephalography. Neuroimage. 2011;57(4):1348-57.

27. Brodbeck V, Lascano AM, Spinelli L, Seeck M, Michel CM. Accuracy of EEG source imaging of epileptic spikes in patients with large brain lesions. Clin Neurophysiol. 2009;120(4):679-85.

28. Brodbeck V, Spinelli L, Lascano AM, Pollo C, Schaller K, Vargas MI, et al. Electrical source imaging for presurgical focus localization in epilepsy patients with normal MRI. Epilepsia. 2010;51(4): 583-91.

29. Michel CM, Lantz G, Spinelli L, De Peralta RG, Landis T, Seeck M. 128-channel EEG source imaging in epilepsy: clinical yield and localization precision. J Clin Neurophysiol. 2004;21(2):71-83.

30. Siniatchkin M, Groening K, Moehring J, Moeller F, Boor R, Brodbeck V, et al. Neuronal networks in children with continuous spikes and waves during slow sleep. Brain J Neurol. 2010;133(9): 2798-813.

31. Lantz G, Spinelli L, Seeck M, de Peralta Menendez RG, Sottas CC, Michel CM. Propagation of interictal epileptiform activity can lead to erroneous source localizations: a 128-channel EEG mapping study. J Clin Neurophysiol. 2003;20(5):311-9.

32. Allen PJ, Josephs O, Turner R. A method for removing imaging artifact from continuous EEG recorded during functional MRI. Neuroimage. 2000;12(2):230-9.

33. Allen PJ, Polizzi G, Krakow K, Fish DR, Lemieux L. Identification of EEG events in the MR scanner: the problem of pulse artifact and a method for its subtraction. Neuroimage. 1998;8(3):229-39.

34. Bell AJ, Sejnowski TJ. An information-maximization approach to blind separation and blind deconvolution. Neural Comput. 1995;7(6):1129-59.

35. Alkawadri R, So NK, Van Ness PC, Alexopoulos AV. Cingulate epilepsy: report of 3 electroclinical subtypes with surgical outcomes. JAMA Neurol. 2013;70(8):995-1002.

36. Catana C, Drzezga A, Heiss WD, Rosen BR. PET/MRI for neurologic applications. J Nucl Med. 2012;53(12):1916-25.
37. Salamon N, Kung J, Shaw SJ, Koo J, Koh S, Wu JY, et al. FDGPET/MRI coregistration improves detection of cortical dysplasia in patients with epilepsy. Neurology. 2008;71(20):1594-601.

38. Martinez-Moller A, Nekolla SG. Attenuation correction for PET/ MR: problems, novel approaches and practical solutions. Z Med Phys. 2012;22(4):299-310.

39. Bailey DL, Barthel H, Beuthin-Baumann B, Beyer T, Bisdas S, Boellaard R, et al. Combined PET/MR: Where are we now? Summary report of the second international workshop on PET/ MR imaging April 8-12, 2013, Tubingen, Germany. Mol Imaging Biol. 2014;16(3):295-310.

40. Dickson JC, O'Meara C, Barnes A. A comparison of CT- and MRbased attenuation correction in neurological PET. Eur J Nucl Med Mol Imaging. 2014;41(6):1176-89.

41. Genetti M, Grouiller F, Vulliemoz S, Spinelli L, Seeck M, Michel $\mathrm{CM}$, et al. Noninvasive language mapping in patients with epilepsy or brain tumors. Neurosurgery. 2013;72(4):555-65; discussion 65.

42. Lascano AM, Grouiller F, Genetti M, Spinelli L, Seeck M, Schaller $\mathrm{K}$, et al. Surgically relevant localization of the central sulcus with high-density somatosensory-evoked potentials compared with functional magnetic resonance imaging. Neurosurgery. 2014;74(5):517-26.

43. Jacobs J, Kobayashi E, Boor R, Muhle H, Stephan W, Hawco C, et al. Hemodynamic responses to interictal epileptiform discharges in children with symptomatic epilepsy. Epilepsia. 2007;48(11): 2068-78.

44. Noth U, Laufs H, Stoermer R, Deichmann R. Simultaneous electroencephalography-functional MRI at $3 \mathrm{~T}$ : an analysis of safety risks imposed by performing anatomical reference scans with the EEG equipment in place. J Magn Reson Imaging JMRI. 2012;35(3):561-71.

45. Luo Q, Glover GH. Influence of dense-array EEG cap on fMRI signal. Magn Reson Med. 2012;68(3):807-15.

46. Chu CJ. High density EEG - what do we have to lose? Clin Neurophysiol. 2014;126(3):433-4. 